\title{
A Modular Framework for Ambient Health Monitoring
}

\author{
Daniel Burmeister, Andreas Schrader \\ Institute of Telematics (Ambient Computing Group) \\ University of Lübeck \\ Lübeck, Germany \\ danielburmeister@web.de, schrader@itm.uni-luebeck.de
}

\author{
Darren Carlson \\ School of Computing \\ National University of Singapore \\ Republic of Singapore \\ carlson@comp.nus.edu.sg
}

\begin{abstract}
Ambient Health Monitoring is becoming increasingly important for supporting proactive self-monitoring as part of a healthy lifestyle and as an enabler of appropriate healthcare services in Ambient Assisted Living (AAL). However, the heterogeneity of available context sources and AAL infrastructure components hinders the design of holistic systems. In this paper, we describe a comprehensive modular system approach for integrating heterogeneous context sources, including: stationary sensor networks in AAL infrastructure; wireless medical device sensors; embedded mobile device sensors; as well as virtual sensors. We outline the potential use cases of the system by presenting a prototype lifelogging application for Android, which dynamically integrates several sensor types into a personal health record, with a special focus on activity recognition. The application also demonstrates the usage of gamification methods as a persuasive means of enhancing the intrinsic motivation of users towards a personalized healthy lifestyle.
\end{abstract}

Keywords-Activity Recognition; Personal Health Record; Ambient Health Monitoring; SmartAssist; Ambient Dynamix

\section{Introduction}

Worldwide, human life expectancy has been growing for many years. This fortunate development is made possible, in large part, by significant improvements in advanced health monitoring technologies and related treatments. Whereas in the past, assessment of vital parameters was restricted to a doctor's office or a clinical environment, recent advances in sensor technologies, mobile devices and wireless networks have enabled the proliferation of continuous Ambient Health Monitoring (AHM) at home and on the move.

Longer lifetimes creates challenging demands for the organization of daily life of elderly people with degrading mental and physical abilities, and will impose significant financial and organizational burdens on the healthcare systems of societies confronted with this demographic change. Ambient Assisted Living (AAL) research approaches these challenges by providing technical healthcare solutions, which are often based on monitoring health-related data. AHM can be used proactively as a means of encouraging healthy life strategies in younger people (e.g., Quantified Self Movement [1]) and for fulfilling the increasing demands by the elderly population for support mechanisms that enable an autonomous, active and fulfilling lifestyle.

AAL projects are often focused on developing high-tech infrastructures that replace missing or fading human abilities (e.g., eating) with (semi-) automated technical services (e.g., feeding robots). Moreover, many AAL projects focus on specific aspects of service provisioning and develop dedicated solutions for those. Examples include sophisticated solutions for dedicated sensing tasks, like in the DiaTrace project [2], which combines activity detection, meal photographs, blood sugar monitoring, etc. into a dedicated nutrition support system.

Unfortunately, most of these approaches are not interoperable, require significant technical infrastructure and maintenance, and usually only fulfill service aspects that have been planned within the scope of the respective projects. There are first attempts to deal with the interoperability challenges of AAL infrastructures by creating middleware and reference architectures with standardized protocols [3], but these usually don't focus on monitoring aspects per se.

Therefore, the development of comprehensive AHM technologies is faced with significant challenges. First, the associated logging infrastructure must support a plethora of sensor device types, including sensor networks in fixed infrastructures; mobile sensors on portable devices; wirelessly connected medical devices; and virtual sensors interpreting local and remote data. Second, the syntax, semantics and access methods of rich contextual information may not be known beforehand, due to the rapid development and deployment of diverse data sources. AHM technology should, therefore, be able to dynamically integrate newly available data sources at runtime and should also be able to flexibly adapt the system to the availability of these new sensor types.

In this paper, we present a holistic approach for AHM that supports the above requirements. Our approach includes a lifelogging mobile application that dynamically integrates diverse context data from wireless sensor networks; commodity health devices; and activity recognition sensors on common smartphone platforms. We evaluate the system through user surveys and a prototype application evaluation that uses gamification techniques to support self-intrinsic user motivation. 


\section{System Architecture}

We base our proposal on the SmartAssist system architecture, which was previously developed at our Institute within the framework of a German BMBF-sponsored AAL project [4]. SmartAssist provides an open platform for the creation of context-aware AAL services that are connected to the social network of elderly, both at home and on the move. The SmartAssist infrastructure consists of an in-home wireless sensor network; a central data server and processing platform; a web based service portal; and a mobile software platform (described below). Users can subscribe to and use AAL services, define peers in their social networks (patrons) that will be informed about significant variations of their vital data, and much more (see [5] for details).

To support seamless context-aware services outside SmartAssist households, we also developed a mobile infrastructure called Ambient Dynamix [6], which is a community-based approach for context-aware computing in which advanced sensing and acting capabilities are deployed on-demand to mobile devices as plug-ins. Dynamix runs as lightweight background service on the user's mobile device, leveraging the device itself as a sensing, processing and communications platform. Dynamix comes with a growing collection of ready-made plug-ins and provides open software developments kits (SDKs) and a scalable repository architecture, which enable $3^{\text {rd }}$ party developers to quickly create and share new plug-in types with the community. Both native apps and Web apps can request context support from a local Dynamix instance using simple application programming interfaces (APIs). Dynamix automatically discovers, downloads and installs the plug-ins needed for a given sensing or acting task. When the user changes environments, new or updated plug-ins can be deployed to the device at runtime, without the need to restart the application or framework. For details on the Dynamix approach, please see [7].

In this work, we leverage Dynamix as the basis for developing lifelogging applications for mobile devices. In order to demonstrate the feasibility of this approach, we have developed a prototype lifelogging application supporting the following sensor types:

\section{Sensor Network Plug-ins}

We developed a dedicated plug-in that enables applications to easily access the data obtained within the wireless sensor network in SmartAssist households. The plug-in obtains user profile and sensor values through the REST-APIs of the SmartAssist server. This principle can be applied to any sensor networks that provide a Web-based proxy.

\section{Medical Device Plug-ins}

We also developed a number of example plug-ins for measuring vital parameters from common medical devices connected directly to the user's device (e.g., via Bluetooth) or via an Internet connection to a data provider's server interface. These plug-ins include:

- $\quad$ Sleep State - This plug-in enables apps to discover a variety of sleep time, phase and quality information provided by a Zeo Mobile Sleep Management device [8] connected via Bluetooth.

- Heart-Rate Biotelemetry - This plug-in delivers heart-rate, step-count and user speed information from a Zephyr Hx fitness-tracking device [9].

- Withings - This plug-in provides weight, fat percentage, pulse and height from a Withings WiFi Digital Body Scale and/or Withings blood pressure monitor [10].

See [7] for a list of health-related Dynamix plug-ins, including sound pressure level, air quality, ambient light, and more.

\section{Virtual Sensor Plug-ins}

Besides vital data obtained from medical devices, the observation of user activities and mobility patterns are equally important for the determination of the user's health situation. Therefore, we created an activity recognition plug-in as a virtual sensor type for Dynamix. Commercial systems such as myVitali [11] or Nike+ Fuelband [12] already use accelerometer data for measuring the user's physical activity. Current generation smartphones also provide the sensor features and processing power necessary to perform single activity recognition directly within the phone [13].

\section{A. Relevant Types of Activity}

To determine relevant activities in terms of User-CenteredDesign [14], a user survey of 9 people between the ages of 22 and 75 was carried out [15]. As a result, the following activities of daily living were considered to be most relevant for activity recognition: walking, running, sitting, standing, cycling, climbing up stairs and climbing down stairs.

As a first attempt, we developed a simple Pedometer plugin that uses the device's inbuilt accelerometer to accurately detect step events and associated step force, which can be used to disambiguate between running and walking [7]. For simple step detection, algorithms based on accelerometer data can achieve sufficient accuracy [13]; however, for the detection of daily living activities, additional sensors (e.g., gyroscope) and algorithmic techniques are necessary.

\section{B. Activity Detection using Trained Classifier}

To provide a foundation for advanced activity detection, a training classifier was constructed. In order to collect sufficient training data, 3 subjects were instructed to perform each aforementioned activity (e.g., walking, running, sitting) over a period of 120 seconds, while wearing the smartphone in their pocket. The following features were extracted (per axis) from the data collected from the gyroscope and accelerometer: mean, standard deviation, energy, correlation coefficient. The suitability of these features has already been demonstrated [16]. Since the movement of each activity has a different duration, the concept of sliding windows with different window sizes of 1, 2 and 3 seconds was applied to the feature extraction process.

The obtained data were imported in the data mining tool WEKA [17] and used to train 12 different classifiers and to examine their suitability using a 10-folded cross-validation, 
including the k-Nearest-Neighbor, Naive Bayes and the C4.5 classifier. These algorithms are also listed among the 10 best classifiers in data mining area [18]. Taking account of a sufficiently high accuracy of each activity, the C4.5 algorithm [19] with a window size of 2 seconds has been identified as best suited for recognizing the intended activities. These results were integrated into the Dynamix Activity Recognition plug-in. By default, the trained C4.5 classifier is used, but it can be replaced by other classifiers, even at runtime [15].

\section{Prototype Lifelogging Application}

To investigate the validity of our approach, we developed a prototype lifelogging Android app based on Dynamix. This section describes the target groups, usage contexts, design goals and the lifelogging app itself.

\section{A. Target Groups \& Usage contexts}

The design of the lifelogging app was based on the results of the previously mentioned user study [15]. As such, the following usage contexts were considered most relevant to the users: 1) Visualization of vital parameters - This context of use was considered the most fundamental since users want to measure and view their vital signs to assess their current health state; 2) Sports - Athletes use the application before, during and shortly after sportive activities in order to evaluate their performance; 3) Slim - In this context, people observe their weight loss progress, paying special attention to physical activity and energy balance; and, 4) Healthcare Support Within this context, the measured data of the application is not primarily relevant to the user, but can be basis for a notification of a healthcare professional in case of deviations from standard values.

\section{B. Design Goals}

In addition to the above design contexts, the system should be designed for both novice and expert users. In particular, elderly people should be supported with simplified user interfaces, self-describing capabilities [20] and integrated help functionality. Importantly, simple measurement of vital signs should be possible and derived data should be visualized coherently using simplified information hierarchies. In addition, gamification methods (e.g., goals, scores, alerts, achievements, etc.) [21] can be used for generating intrinsic user motivation [22]. These techniques should be introduced in relation to the usage context, to address users' needs without overwhelming them. Users should be able to set goals themselves and monitor their progress. Automatically created and manually configured challenges should be supported. Currently measured vital signs should display the user's health status through an aggregated value that is comprehensible at a glance.
Detailed views should enable more information and trend indications.

\section{Android Application}

Using the above design goals, we developed a Dynamixbased Android app that provides comprehensive lifelogging features. The app provides several interfaces, as shown in Fig. 1. Dynamix plug-ins can be loaded and activated on demand and are used to measure respective health values using inbuilt and external sensors (a). If users do not have a particular sensor, they can enter values manually. A reminder can be assigned to each vital parameter for daily or weekly measuring. The user's health status is represented by an aggregated value that is displayed in the main screen of the application (b). This value, known as the fitness factor, represents the mean deviation of currently measured vital signs from standard values.

In addition to the challenges provided by the application, users can set goals themselves by indicating the activity, a target date and the amount (c). Measured values are displayed in a real-time view (d). Tilting the device changes the view into a type-dependent course representation, in which the user can navigate using a swipe gesture that changes the hierarchy of representation (e-f).
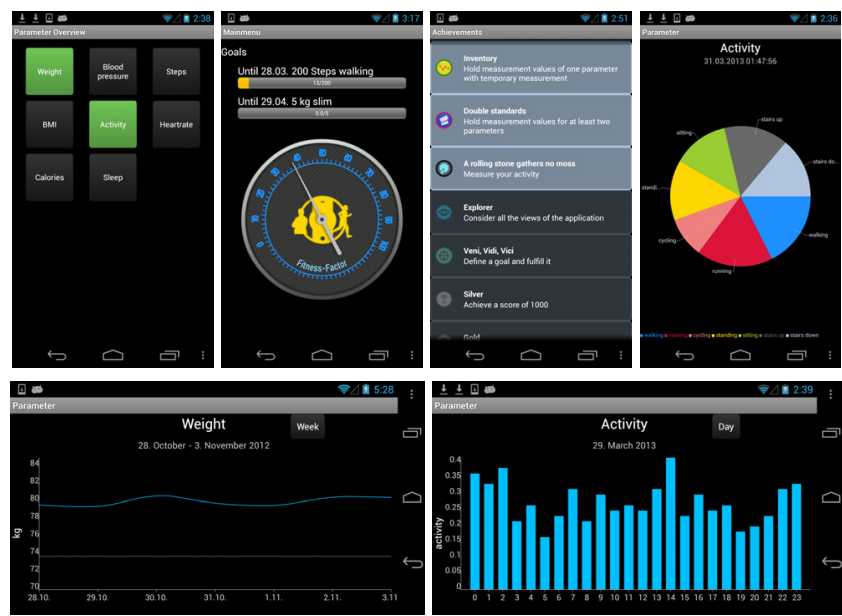

Fig. 1. Example Screenshots from the Lifelogging App (left to right): (a) Available Plug-ins (b) Main View (c) Earned and open Achievements (d) Pie

Chart (Activity) (e) Continuous Graph (Weight) (f) Bar Chart (Activity)

Users can import an existing SmartAssist user profile or create a new one. The profile is used to calculate standard values based on biometric data, to specify a patron to be notified per SMS in case of vital parameter deviation, and to load a set of personalized context-aware achievements. Using the key-value principle for persistence of the PHR enables the dynamic addition of further parameters and structured storage of values, depending on their name and measuring frequency.

\begin{tabular}{c|c|c|c|c|c|c|c|} 
& Walking & Running & Cycling & Standing & Sitting & Stairs up & Stairs down \\
\hline Sensitivity & $86.84 \%$ & $94.74 \%$ & $90.43 \%$ & $100 \%$ & $100 \%$ & $74.14 \%$ & $92.46 \%$ \\
Specificity & $97.61 \%$ & $99.24 \%$ & $98.43 \%$ & $100 \%$ & $100 \%$ & $94.66 \%$ & $98.94 \%$ \\
Precision & $93.75 \%$ & $82.28 \%$ & $96.04 \%$ & $92.39 \%$ & $96.04 \%$ & $93.06 \%$ & $78.48 \%$ \\
\hline
\end{tabular}




\section{Evaluation}

In our preliminary evaluation, we validated the activity recognition plug-in accuracy and determined the application's usability. These results are described next.

\section{A. Activity Recognition}

The classifier's accuracy was evaluated by asking the same 3 subjects to perform the trained activities over a period of 60 seconds under the same conditions as the training phase. Every second, the activity detected by the plug-in was recorded into a confusion matrix. From this, we calculated sensitivity (percentage of correctly true-positive classification), specificity (percentage of correctly falsenegative classification), and precision (percentage of correctly true-positive classified activities in the same class). An average precision of $90 \%$ was achieved for walking, cycling, standing and sitting. The stairs down activity was the most difficult to recognize ( $78 \%$ precision). A closer look at the confusion matrix shows that the stairs up activity had the lowest sensitivity, which impaired the detection of other activities.

\section{B. Application}

The lifelogging application's usability was evaluated by interviewing the same 9 people participating in the survey. While the application's interface was broadly viewed as helpful, tilting the device to change between the currently measured view and the course representation view was not obvious. In addition, the calculation of the fitness factor needs to be more transparent. A more detailed personalization of the application was considered desirable by several people. In this case, previous medical records or ongoing monitoring could be used to help calculate standard values for various health parameters. Furthermore, vital signs such as blood sugar could be added with appropriate measuring devices. Finally, in the opinion of several interviewees, goal setting or achievement attainment can be enhanced through the integration of social network features (e.g., achievement notifications and/or posting).

\section{Conclusions}

In this work, we presented a personalized AAL healthcare system that dynamically integrates data from both inbuilt and remote sensors within a lifelogging application. The system combines existing Dynamix features with a custom-built Activity Recognition plug-in that is capable of accurately detecting common activity types. The system was evaluated using an example Android-based app that uses Dynamix features and gamification techniques to promote ongoing self monitoring of health related information.

We are focused on several areas of future work. Currently, we are developing additional related Dynamix plug-ins, such as virtual sensors for social activities and camera-based detection of gait and seating positions. We also recently extended the Dynamix framework to support browser-based Web applications [23]. As such, we are investigating how lifelogging apps can be easily created using a combination of
Web techniques and powerful JavaScript-based visualization tools.

This project was funded by the German Federal Ministry of Education and Research. BMBF Grant Number: 16KT094. The system is available as open source and we invite the research community to join the process.

\section{References}

[1] Quantified Self, http://quantifiedself.com/

[2] Bieber, G. und Thom, A. „DiaTrace - Neuartiges Assistenz-System für die Gesundheitsprävention zur Nahrungsaufnahme und Bewegungserfassung.", in Proceedings of 1st German AAL Congress, Berlin, Germany, January 2008.

[3] UniversAAL project, http://www.universaal.org

[4] D. Carlson, P. Rothenpieler, and A. Schrader, "An open infrastructure and platform for AAL services," to appear in International Conference on Distributed, Ambient and Pervasive Interactions (DAPI'13). Las Vegas, USA: HCI International 2013, July 2013.

[5] SmartAssist Project, http://www.smartassist.de

[6] D. Carlson and A. Schrader, "Dynamix: An Open Plug-and-Play Context Framework for Android," in 3rd International Conference for Industry and Academia (Internet of Things 2012), Wuxi, China, October 2012, pp. 24-26.

[7] Ambient Dynamix Project, http://ambientdynamix.org

[8] ZEO Sleep Manager, http://www.myzeo.com/sleep/

[9] Zephyr Heart-rate Monitor, http://www.zephyr-technology.com/

[10] Withings Scale and Blood Pressure Monitor, http://www.withings.com/

[11] T. Ulmer, "myVitali - modulares Assistenzsystem zur Gesundheitsprävention und Rundumversorgung," in Proceedings of 2nd German AAL Congress, Germany, January 2009.

[12] Nike+ Fuelband, http://www.nike.com/us/en_us/c/nikeplus-fuelband

[13] T. S. Saponas, J. Lester, J. Froehlich, J. Fogarty, and J. Landay, "ilearn on the iphone : Real-time human activity classification on commodity mobile phones," University of Washington CSE Tech Report UWCSE080402, pp. 1-4, 2008.

[14] D. A. Norman and S. W. Draper, User Centered System Design; New Perspectives on Human-Computer Interaction. Hillsdale, NJ, USA: L. Erlbaum Associates Inc., 1986.

[15] D. Burmeister, Aktivitätserkennung für Ambient Health Monitoring. Master's Thesis at the University of Luebeck, Germany, 2012.

[16] L. Bao and S. S. Intille, "Activity recognition from user-annotated acceleration data," Pervasive Computing, vol. LNCS 3001, pp. 1-17, 2004.

[17] M. Hall, E. Frank, G. Holmes, B. Pfahringer, P. Reutemann, and I. H. Witten, "The weka data mining software: an update," SIGKDD Explor. Newsl., vol. 11, no. 1, pp. 10-18, Nov. 2009.

[18] X. Wu, V. Kumar, J. Ross Quinlan, J. Ghosh, Q. Yang, H. Motoda, G. J. McLachlan, A. Ng, B. Liu, P. S. Yu, Z.-H. Zhou, M. Steinbach, D. J. Hand, and D. Steinberg, "Top 10 algorithms in data mining," Knowl. Inf. Syst., vol. 14, no. 1, pp. 1 - 37, December 2007.

[19] R. J. Quinlan, C4.5: programs for machine learning. San Francisco, CA, USA: Morgan Kaufmann Publishers Inc., 1993.

[20] D. A. Norman, The Psychology of Everyday Things. New York: Basic Books, 1988, pp. 9ff.

[21] J. E. Barbuto and R. W. Scholl, "Motivation sources inventory: development and validation of new scales to measure an integrative taxonomy of motivation," in Psychological Reports. Ammons Scientific, 1998, vol. 82 i3, pp. 1011 - 1022.

[22] G. Zichermann and C. Cunningham, Gamification by Design Implementing Game Mechanics in Web and Mobile Apps. O'Reilly Media, Inc., 2011.

[23] D. Carlson, B. Altakrouri, and A. Schrader. "Reinventing the Share Button for Physical Spaces", in: Percom'2013, Demo Session at IEEE International Conference on Pervasive Computing and Communication (PerCom 2013), San Diego, California, USA, March 18-22, 2013. 